\title{
Particle velocity profile in an inclined rotating drum
}

\author{
$P$. Widhate, H. P. Zhu, Q. H. Zeng, and $K . J$. Dong \\ School of Engineering, Western Sydney University, Locked bag 1797, Penrith, NSW 2751, Australia
}

\begin{abstract}
Various experimental and numerical studies have been carried out to study the velocity profiles of the particles inside horizontal rotating drums, but little emphasis has been laid on inclined rotating drums, though these drums are extensively used in granular process industries. In this study, velocity profiles of the particles in a rotating drum with $0^{\circ}, 5^{\circ}, 10^{\circ}$, and $15^{\circ}$ inclinations have been studied by using the discrete element method. It was found that at the locations of the drum with the volumetric fill in the range of $0.2-0.8$, the inclined rotating drum has a similar variation of velocity with the radial height to the horizontal rotating drum. However, the variation of the average particle velocity along the length of the drum differs for horizontal and inclined drums. Furthermore, for the inclined rotating drum, the average velocity increases with the increase in the volumetric fill.
\end{abstract}

\section{Introduction}

Simple geometry and multiple applications such as drying, mixing, segregation and heat conduction have inspired many researchers from the mechanical, chemical, pharmaceutical and mining engineering fields to study the dynamics of the particle flow inside rotating drums [1]. The velocity profile of particles in rotating drums is one of the key properties considered in these studies. It has been found that for monodisperse particles, the profile of transverse velocity (component of velocity in the direction perpendicular to the bed surface of the drum) in the radial plane of a rotating drum follows a linear trend with the bed height. The profile of streamwise velocity (component of velocity in the direction parallel to the bed surface) differs as it behaves linearly, but changes its slope after achieving a certain bed height [1]. The change in the slope of the streamwise velocity distribution results in the formation of two different flow regions inside the rotating drum. The variation of the ratio of the magnitude of streamwise velocity to its maximum on the bed surface along the radial direction follows the second-degree polynomial trend, which is also true for inner layers of the particle bed [2]. Further, in Ref. [3], normalized velocity profiles were used to evaluate the different flow regimes inside a rotating drum. It was found that the normalized velocity with high peaks could define the slumping regime. In cascading and cataracting regimes, the normalized velocity almost remains linear, and increases with the increase in angular velocity of the drum. In addition, the average velocity of particles also increases with an increase in drum angular velocity or with a decrease in the volumetric fill of particles [3].
Therefore, the velocity profile is a very important property in understanding the particle flow in rotating drums.

While most of the studies have been conducted to examine the velocity profile for rotating drums with horizontal axis of rotation, little emphasis has been given to study inclined rotating drums. It is important to understand the dynamics of the particle flow in an inclined rotating drum as such drums are widely used in industries. In this study, the discrete element method (DEM) was used to study the velocity profile in an inclined rotating drum. Four angles of inclination for the drum ranging from $0^{0}$ to $15^{0}$ were considered and the velocity profile of particles was examined in detail.

\section{Simulation method and conditions}

In this study, the EDEM software was used to simulate the particle flow in an inclined rotating drum. The software is based on the DEM. This method was first proposed by Cundall and Strack and has been developed later to study the dynamics of the particles in various applications $[1,4]$. The motion of the particles inside a rotating drum can be described by Newton's second law of motion $[1,4]$. In the case of macro-sized particles, governing equations for translational and rotational motion of the particles can be given by

$$
\begin{gathered}
m_{i} \frac{d \boldsymbol{v}_{i}}{d t}=\sum_{j} \boldsymbol{F}_{i j}^{c}+\boldsymbol{F}_{i}^{g} \\
I_{i} \frac{d \boldsymbol{\omega}_{i}}{d t}=\sum_{j} \boldsymbol{M}_{i j}
\end{gathered}
$$




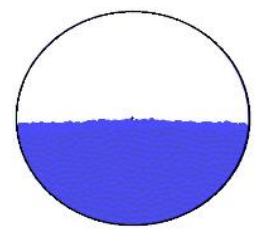

(a)

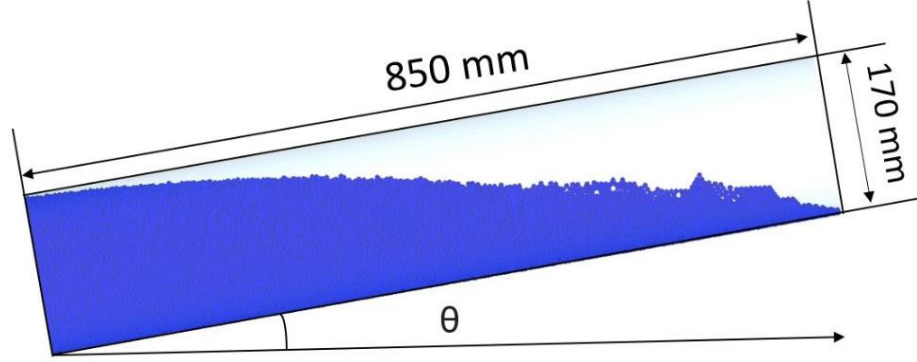

(b)

Fig.1. (a) Initial radial view; and (b) axial view of the drum at the steady state for $\theta=10^{\circ}$, where $\theta$ is the angle of inclination.

where $\boldsymbol{v}_{i}$ and $\boldsymbol{\omega}_{i}$ are the translational and angular velocities of particle $\mathrm{i}$, respectively, $m_{i}$ and $I_{i}$ are the mass and moment of inertia of particle i, $\boldsymbol{F}_{i}^{g}$ is the gravitational force acting on particle $\mathrm{i}, \boldsymbol{F}_{i j}^{c}$ and $\boldsymbol{M}_{i j}$ are the contact force and torque acting on particle $\mathrm{i}$ by particle $\mathrm{j}$ or drum wall, respectively. There are a few models which have been popularly used to calculate the particle-particle and particle-wall interaction forces. In this study, the Hertz-Mindlin no-slip model was used for contact forces. Detailed discussion on the force models can be seen elsewhere, for example, in [5]. Eqs. (1) and (2), which are based on the forces and torques between particles, can be solved numerically. Thus, the trajectories, velocities and transient forces of all the particles in a system can be determined.

Table 1. Material properties and parameters used in the simulations

\begin{tabular}{|c|c|}
\hline Properties & Parameter \\
\hline Fill degree & $50 \%$ \\
\hline Drum diameter & $170 \mathrm{~mm}$ \\
\hline Particle diameter & $5 \mathrm{~mm}$ \\
\hline Particle number & 80000 \\
\hline Density of particles & $1430 \mathrm{~kg} / \mathrm{m}^{3}$ \\
\hline $\begin{array}{l}\text { Coefficient of } \\
\text { sliding friction }\end{array}$ & 0.16 \\
\hline $\begin{array}{l}\text { Coefficient of } \\
\text { rolling friction }\end{array}$ & $\begin{array}{l}0.0000722 \text { (particle-drum), } \\
0.0000594 \text { (particle-particle) }\end{array}$ \\
\hline Young's modulus & $\begin{array}{l}2.84 \times 10^{9} \mathrm{~Pa} \text { (particle), } 2.2 \times 10^{9} \\
\mathrm{~Pa}(\text { drum })\end{array}$ \\
\hline Poisson's ratio & 0.35 (particle, drum) \\
\hline $\begin{array}{l}\text { Coefficient of } \\
\text { restitution }\end{array}$ & $\begin{array}{l}0.80 \text { (particle-drum), } 0.88 \\
\text { (particle-particle) }\end{array}$ \\
\hline
\end{tabular}

An inclined rotating drum with diameter $\left(D_{d}\right) 170$ $\mathrm{mm}$ and length $\left(L_{d}\right) 850 \mathrm{~mm}$ (i.e., $L_{d} / D_{d}=5$ ) was used in the simulation to investigate the effect of angle of inclination on particle velocity profile. Four simulations with angles of inclination $0^{\circ}$ (horizontal), $5^{\circ}, 10^{\circ}$ and $15^{\circ}$ have been carried out. In each simulation, 80,000 particles of $5 \mathrm{~mm}$ diameter were generated and placed in the drum. The material properties for the particles and drum were the same as those used in Elskamp et al. [5] and are listed in Table 1. A volumetric fill of approximately $50 \%$ across the axial length of the drum has been achieved in each case (Fig. 1(a)). After the particles settled down, the system was simulated with an angular speed of 30 RPM until a steady state was achieved. In this work, when the average properties considered are almost constant, the particle flow was considered to be at a steady state. The time found when the steady state was achieved for all cases is $\mathrm{t}=30 \mathrm{~s}$. Later, the drum was divided into a number of cylindrical subsections from $\mathrm{L}=0$ to 850 $\mathrm{mm}(\mathrm{L}=0 \mathrm{~mm}$ was considered as the lower end of the drum and $\mathrm{L}=850 \mathrm{~mm}$ the higher end.). The cylindrical subsections are perpendicular to the drum axis, with thickness of $15 \mathrm{~mm}$ and diameter equivalent to that of the drum. The properties such as volumetric fill, velocity profile, and average velocity were then calculated at each subsection.

\section{Results and discussion}

\subsection{Volumetric fill}

In the beginning of rotation of the inclined rotating drum, particles tend to move towards the lower end due to the gravitational force acting on the particles in the axial direction. Hence, in the process, particles are accumulated in the lower part of the drum leading to an increase in this part and a decrease in the upper part in the volume occupied by particles (Fig. 1(b)). To quantify the phenomenon, volumetric fill at the steady state has been considered. To determine the volumetric fill at a defined location, the volume occupied by the particles in the cylindrical subsection for the location was first calculated, and the value obtained was then divided by the total volume of the cylindrical subsection. The values of the volumetric fill at locations with a distance of $\mathrm{L}=50 \mathrm{~mm}$ in between were calculated from $\mathrm{L}=0$ to $850 \mathrm{~mm}$.

Fig. 2 shows the variation of the volumetric fill along the length of the drum for different angles of inclination. It can be observed that in the case of $0^{\circ}$ inclination, the volumetric fill nearly equals to 0.5 , which is similar to that obtained in previous studies for horizontal rotating drums. In the cases of inclined rotating drum, the volumetric fill decreases with the length of the drum. With an increase in the angle of inclination, it increases in the lower half of the drum (i.e., from $\mathrm{L}=0$ to $425 \mathrm{~mm}$ ) and decreases in the upper 
half (i.e., $\mathrm{L}=425$ to $850 \mathrm{~mm}$ ). The reason why this phenomenon occurs is because more particles intend to move towards the lower end of the drum for a higher inclination.

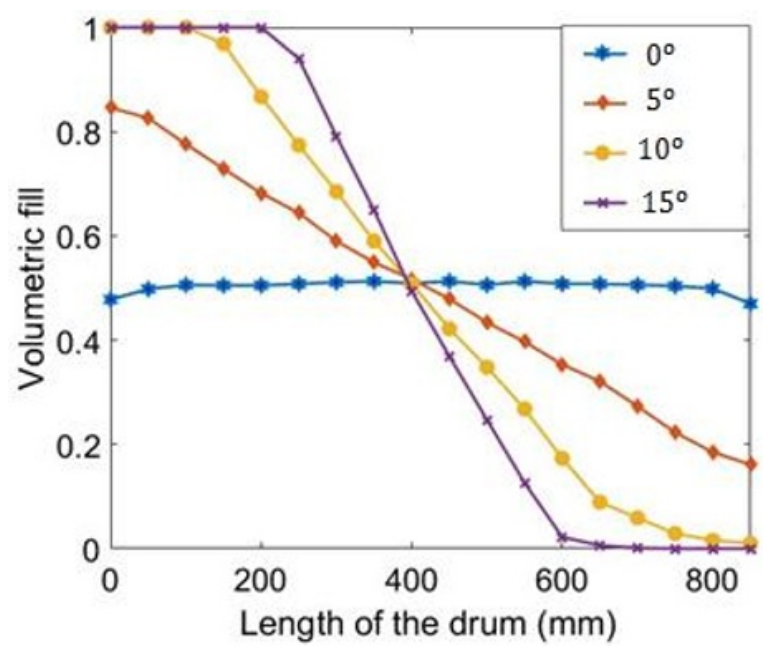

Fig. 2. Volumetric fill along the length of the drum for $\theta=$ $0^{\circ}, 5^{\circ}, 10^{\circ}$ and $15^{\circ}$.

\subsection{Velocity profile}

Fig. 3 shows the velocity distribution of particles on the cross section of the drum at $\mathrm{L}=425 \mathrm{~mm}$ for $10^{\circ}$ inclination. For the brevity of description, a coordinate system on the section was defined. In the system, the $\mathrm{X}^{*}$ axis is along the bed surface, the $\mathrm{Y}^{*}$ axis is the perpendicular bisector of the surface, and $\mathrm{X}^{*}=\mathrm{Y}^{*}=0$ is at the wall of the drum. It can be observed that the magnitude of the velocity in the $\mathrm{X}^{*}$ direction is higher than the one near the wall. The phenomenon exists at other locations as well, and is similar to that for a horizontal rotating drum [1].

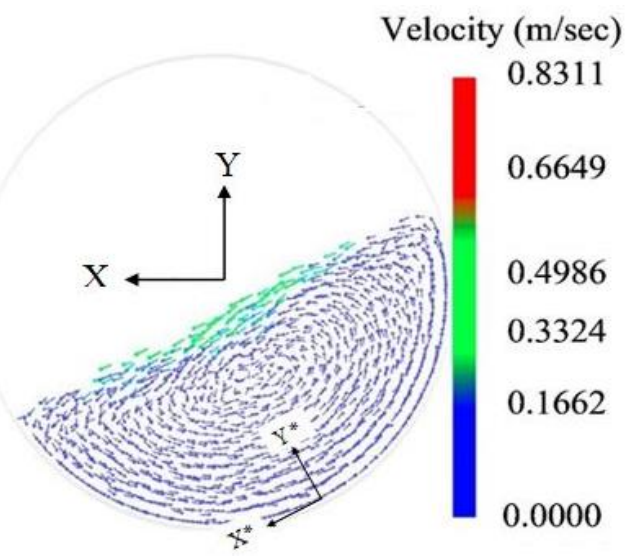

Fig. 3. Velocity distribution of particles on the cross section of the drum at $\mathrm{L}=425 \mathrm{~mm}$ for $\theta=10^{\circ}$.

To get a deeper insight view of the velocity profile in the inclined rotating drum, the velocity in the $\mathrm{X}^{*}$ direction, $\mathrm{U}_{\mathrm{X}^{*}}$, along the $\mathrm{Y}^{*}$ axis has been calculated at five locations $\mathrm{L}=25,225,425,625$ and $825 \mathrm{~mm}$ for $0^{\circ}, 5^{\circ}, 10^{\circ}$ and $15^{\circ}$ inclinations. Fig. 4 shows the variation of the velocity $\mathrm{U}_{\mathrm{x}^{*}}$ along the $\mathrm{Y}^{*}$ direction for the five locations. Note that not all locations have been considered for some angles of inclination. For example, for $\theta=0^{\circ}$, only the location at $\mathrm{L}=425 \mathrm{~mm}$ has been included in the figure. This is because other locations have the similar velocity distribution for the horizontal drum. For $\theta=15^{\circ}$, locations $\mathrm{L}=625$ and $825 \mathrm{~mm}$ have not considered as there are very small number of particles at these locations. It can be observed that for the locations where the volumetric fill is within range of $0.2-0.8$, the distribution of $U_{x^{*}}$ follows a similar trend to that for horizontal rotating drum under rolling regime conditions, that is, the velocity linearly increases to a certain bed height, and then increases with a higher gradient. The two segments of the distribution correspond to two different flow regions: surface flow and plug flow regions. When the volumetric fill is higher than 0.8 (at the locations of $25 \mathrm{~mm}$ for $\theta=10^{\circ}$ and $15^{\circ}$ ) or lower than 0.2 (at the locations of $625 \mathrm{~mm}$ for $\theta=10^{\circ}$ and $825 \mathrm{~mm}$ for $\theta=5^{\circ}$ ), the velocity has a linear distribution along the bed height. The reason for the existence of such s profile is different for higher and lower volumetric fills. For high volumetric fills, the relative motion of the particles gets restrained due to less space in the bed. The particles on the cross section for such volumetric fills exhibit the plug flow behavior. In contrast, at the locations with low volumetric fill, there are no many particles. These particles are in the motion similar to that in the surface flow region.

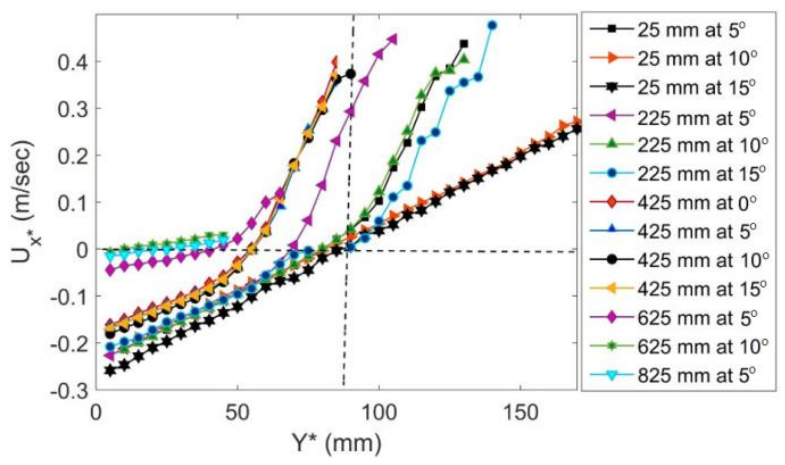

Fig. 4. Distributions of velocity $\mathrm{U}_{\mathrm{x}^{*}}$ along the $\mathrm{Y}^{*}$ axis at different locations for different angles of inclination.

\subsection{Relationship between average velocity and volumetric fill}

Here, the average velocity at a location refers to the average of velocity magnitudes of the particles in the cylindrical subsection for the location. Fig. 5 shows the variation of average velocity along the length of the drum with different angles of inclination. It can be observed that the average velocity for the horizontal drum is almost constant along the length of the drum. The minor peaks observed at both ends of the drum are caused by the wall effect. For the inclined rotating drum, the average velocity is higher, as compared to the horizontal rotating drum, in the lower half (that is, from $\mathrm{L}=0$ to $425 \mathrm{~mm}$ ), and lesser in the top half (from $\mathrm{L}=425$ to $850 \mathrm{~mm}$ ). 
The average velocity is almost constant in the lower part of the inclined drum, where the volumetric fill is high. It then decreases with the length of the drum, which is similar to the trend of the volumetric fill distribution with the drum length. The reason why there is a high velocity at the lower part may be because the wall effect on the movement of particles is strong in the regions with high volumetric fill. Thus, most particles rotate around the drum axis with the rotation of the drum.

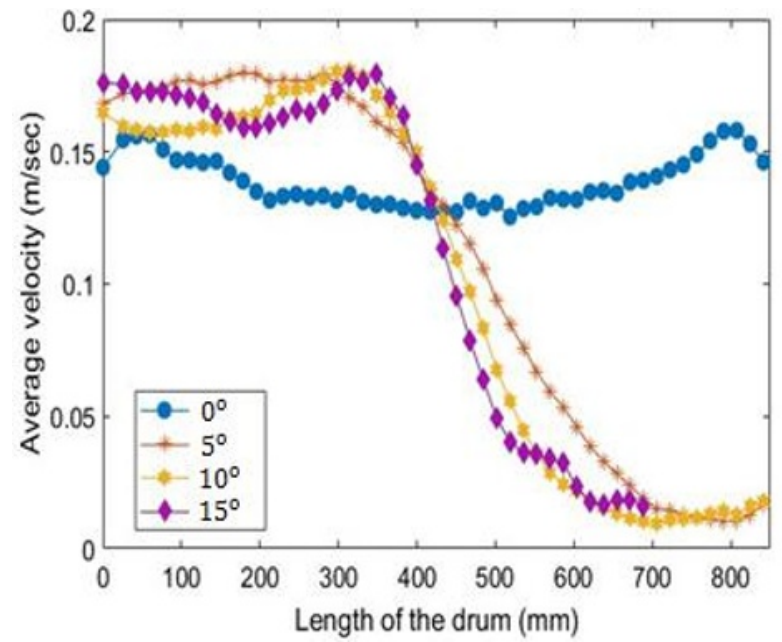

Fig. 5. Variation of the average velocity along the length of the drum for $\theta=0^{\circ}, 5^{\circ}, 10^{\circ}$ and $15^{\circ}$

To understand the relationship between the average velocity and volumetric fill more clearly, Fig.6 shows the dependence of the average velocity on volumetric fill, where the volumetric fill is in the range of $0.2-0.8$. It can be observed that in the range of volumetric fill considered, the average velocity increases with an increase in the volumetric fill. The variation follows a quadratic polynomial pattern. Note that the relationship was established based on the average values of velocity and volumetric fill in the defined subsections. To fully understand the relationship, more points inside the particle bed will be considered, and statistical analysis based on the new data will be carried out in our future work.

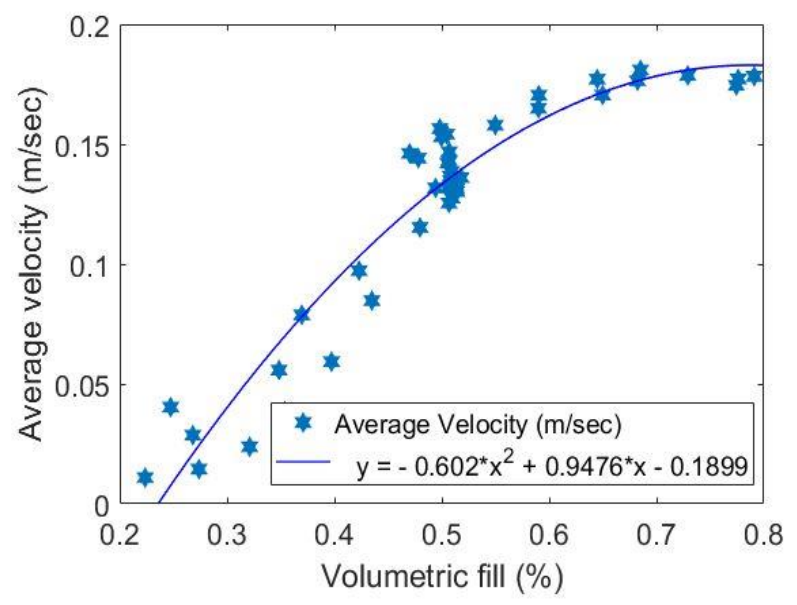

Fig. 6. Dependence of average velocity on volumetric fill.

\section{Conclusions}

In this study, DEM simulations have been carried out with an inclined rotating drum for $0^{\circ}, 5^{\circ}, 10^{\circ}$ and $15^{\circ}$ angles of inclination. It was found that in the cross section of the drum with volumetric fill in the range of $0.2-0.8$, the profiles of the velocity in the bed surface direction along the radial height for the inclined rotating drum are similar to that for the horizontal rotating drum. However, the variations of the average particle velocity along the length of the drum differ comparing the inclined and horizontal rotating drums. The average velocity for the horizontal drum is almost constant along the length of the drum, while it is almost constant in the lower part of the drum and decreases with the length in the higher part for the inclined drum. Considering all angles of inclination, the average particle velocity increases with an increase in the volumetric fill.

\section{Acknowledgment}

The authors are grateful to the Australian Research Council, Jiangsu Industrial Technology Research Institute, and Western Sydney University (ARC IH140100035) for the financial support for this work.

\section{References}

[1] H. Zhu, Z. Zhou, R. Yang, A.B. Yu, Chem. Eng. Sci. 63, 5728-5770 (2008)

[2] E. Alizadeh, O. Dubé, F. Bertrand, J. Chaouki, AIChE J. 59, 1894-1905 (2013)

[3] R. Yang, A.B. Yu, L. McElroy, J. Bao, Powder Technol. 188, 170-177 (2008)

[4] J. Mellmann, Powder Technol. 118, 251-270 (2001)

[5] H. Zhu, Z. Zhou, R. Yang, A.B. Yu, Chem. Eng. Sci. 62, 3378-3396 (2007)

[6] F. Elskamp, H. Kruggel-Emden, M. Hennig, U. Teipel, Granul. Matter 19, 46 (2017) 